Revue d'histoire de l'enfance « irrégulière »

Le Temps de l'histoire

Hors-série | 2001

Histoire et justice, panorama de la recherche

Entre monde judiciaire et philanthropie : la figure du juge-philanthrope au tournant des XIXème et XXème siècles

Pascale Quincy-Lefebvre

\title{
OpenEdition
}

Journals

Édition électronique

URL : http://journals.openedition.org/rhei/435

DOI : $10.4000 /$ rhei.435

ISBN : 978-2-7535-1641-0

ISSN : $1777-540 \mathrm{X}$

Éditeur

Presses universitaires de Rennes

Édition imprimée

Date de publication : 15 novembre 2001

Pagination : 126-139

ISSN : 1287-2431

Référence électronique

Pascale Quincy-Lefebvre, « Entre monde judiciaire et philanthropie : la figure du juge-philanthrope au tournant des XIXème et XXème siècles », Revue d'histoire de l'enfance « irrégulière » [En ligne], Horssérie | 2001, mis en ligne le 31 mai 2007, consulté le 19 avril 2019. URL : http:// journals.openedition.org/rhei/435 ; DOI : 10.4000/rhei.435 


\section{Entre monde judiciaire et philanthropie : la figure du juge- philanthrope au tournant des XIXème et XXème siècles}

Au XIXème siècle, la figure de l'homme de bien, incarné par le notablephilanthrope, se confond fréquemment avec celle de l'homme de loi (juge ou avocat). Fiction littéraire et réalité se rejoignent dans la Comédie bumaine lorsque, à côté de l'homme charitable qu'est le docteur Bénassis, ${ }^{(2)}$ Balzac croque, sous les traits de Popinot, ${ }^{(3)}$ le portrait d'un autre type social : le juge-bienfaiteur des pauvres. Observateur consciencieux de la société de son temps, Balzac rend compte du lien particulier qui unit la représentation de certains états, ou professions, avec celle d'un comportement charitable exemplaire. Il fait dire à son personnage, le docteur Bénassis : "J'ai longtemps hésité à me faire curé, médecin de campagne ou juge de paix. Ce n'est pas sans raison que l'on assemble proverbialement les trois robes noires, le prêtre, l'homme de loi, le médecin. ${ }^{(4) " ~}$

$\mathrm{Au}$ XIXème et au début du XXème siècles, la philanthropie, version volontairement laïcisée de la charité, sert à définir le devoir social du citoyen dans la société libérale née des Lumières et de la Révolution française. Elle pose une morale, renvoie à des pratiques et participe à des formes de sociabilité bourgeoise. Au cours de la deuxième moitié du XIXème siècle, dans un contexte politique et social agité, la philanthropie, par amour de l'humanité et défense d'un ordre jugé menacé, dessine un tissu de relations sociales qui fait du patronage des populations laborieuses par les élites la solution à la misère des masses. Par delà l'héritage des Lumières, les philanthropes au XIXème siècle forment cette population encensée ou conspuée "qui, par des dons, par des écrits, des participations bénévoles, ouvrent au bien des autres hommes et cherchent à soulager leurs maux, que ceux-ci soient matériels, culturels, sociaux ou autres ${ }^{(5)} »$.

Pascale

QuincyLefebvre $^{(1)}$

(1) Historienne,

CHEC, université de

Clermont-Ferrand II

(2) Honoré DE BAL-

ZAC, Le médecin de campagne, Ière édition 1832.

(3) HONORÉ DE

BALZAC, L'Interdiction,

Ière édition 1836.

(4) L'auvre de Balzac, le

Club français du livre, 1962, tome VII, p. 87.

Cité par Catherine

DUPRAT, Usage et pra-

tiques de la philanthropie.

Pauvreté, action sociale et

lien social, à Paris, au

cours du premier XIXème siècle, Paris, Comité

d'histoire de la sécurité sociale, 1997, vol II, p. 1264.

(5) Catherine DUPRAT, op. cit., p. 893. 
L'étude du type social du juge-philanthrope s'insère dans une recherche socio-culturelle du milieu de la magistrature au XIXème et au début du XXème. Sans doute, faut-il d'abord rechercher ce qui, dans la culture des professions juridiques, fait le lien avec l'engagement philanthropique.

\section{Georges Bonjean, magistrat et philanthrope}

Différentes formes d'enquêtes peuvent être menées. Dans le cadre de la présente étude, le choix s'est porté sur l'analyse d'un itinéraire de vie : celui de Georges Bonjean, magistrat parisien, fondateur d'un patronage pour l'enfance en danger moral. L'homme (1848-1918) a été avocat de 1873 à 1876, puis juge au tribunal civil de la Seine, longtemps suppléant puis juge titulaire à partir de 1894 . Sa carrière fut plutôt modeste au plan professionnel. Fils de Louis Bernard Bonjean, catholique libéral, sénateur (1865), Président de la Chambre des requêtes à la Cour de cassation avant 1871, il eut pu nourrir des ambitions plus étendues.

Dans le milieu judiciaire, par la publication en 1895 d'un ouvrage intitulé Enfants révoltés, parents coupables, le nom du magistrat est resté associé à l'introduction de nouvelles pratiques d'enquête dans le service de correction paternelle du tribunal de la Seine, ainsi qu'à des propositions de réformes des articles du Code civil faites au sein de la Société Générale des prisons. Rien de bien extraordinaire, mais l'écho fait à certaines de ses actions est venu de sa réputation de juge-philanthrope auprès de sa hiérarchie ou de ses confrères.

En cette fin de siècle, l'homme est surtout connu pour ses initiatives en direction de l'enfance malheureuse. Parmi ses titres de gloire, figure en bonne place la fondation en 1879, à Paris, de la Société générale de protection pour l'enfance abandonnée ou coupable, avec comme premier vice-président le médecin-sénateur Théophile Roussel, un des pères de la législation sur la protection de l'enfance sous la Troisième République. Parallèlement à cette fonction, le magistrat attacha son nom à l'ouverture de multiples établissements, lieux de placement pour enfants orphelins, délinquants, moralement abandonnés... dans différents départements (Eure, Indre, Oise, Pas-de-Calais). Après 1910 et les procès qui concernèrent deux de ses œuvres pour l'enfance, Georges Bonjean se lança dans 
des réalisations en direction du monde ouvrier (logements, coopératives, organes de presse). Mais la plupart de ces dernières initiatives capotèrent dans les mois ou les années qui suivirent leur mise à exécution.

Le parcours de l'homme est complexe et les interrogations multiples. Deux retiendront plus particulièrement notre attention : derrière la cause de la protection de l'enfance - et plus largement la question sociale - quel est le ressort qui fait courir le magistrat-philanthrope ? Quel rôle les différentes « morales signifiantes », professionnelles, familiales, religieuses, jouentelles ? Partant de l'expérience philanthropique, il conviendra de se poser la question des éventuelles transformations des pratiques judiciaires. ${ }^{(6)}$

\section{Morale et engagements}

Le magistrat-philanthrope est un homme épris de morale : en 1908, il donne à sa revue Le Fanion, le sous-titre d'" organe d'éducation nationale et sociale. Champion des intérêts des Humbles et des Faibles "; l'année suivante, il fonde l'association La Jeune France. Il se met au service du «bien », du "vrai », et du "juste ». Les valeurs défendues par le juge établissent des liens entre vie familiale, engagement patriotique, combat social et exercice d'une profession.

\section{Morale et profession}

Lorsque Georges Bonjean se "lance en philanthropie ", il met en avant sa formation de juriste et sa profession de magistrat comme formes de légitimité et de compétence pour s'occuper de certains problèmes moraux et sociaux. La démarche vise à assurer la promotion de ses "œuvres" dans un milieu philanthropique qui, par la référence à des savoirs et à des compétences, tend à marquer la distance avec la charité traditionnelle, accusée d'incompétence et d'irrationnalité.

Dans la deuxième moitié du XIXème siècle, le temps n'est plus à la philanthropie romantique qui cherchait dans le sentiment les formes de l'action à développer. Désormais s'impose une philanthropie marquée à la fois par la peur du désordre et par la volonté d'apporter des savoirs nouveaux et une plus grande rationalité aux pratiques. Il s'agit parfois d'un "philanthropisme juridique » identifié par Martine Kaluszynski dans
(6) Les cultures philanthropiques participent à un état particulier des cultures pénales et judiciaires et font évoluer la fonction de magistrat en particulier auprès des mineurs. 
(7) Martine KALUS-

ZYNSKI, "A l'origine des politiques pénales en France sous la IIIème République. Un laboratoire de réflexions : la société générale des prisons", dans Colette BEC, Catherine DUPRAT, Jacques Guy PETIT, Jean-Noël LUC (dir.), Philanthropies et politiques sociales (XVIIème-XXème siècles), Paris, Anthropos-historique, 1994, p.142.

(8) Plus tard, il entre en conflit avec celui qui a particulièrement bien incarné cette philanthropie professionnelle : l'inspecteur des prisons et fondateur d'une colonie pénitentiaire privée : Charles Lucas.

(9) Georges BONJEAN, Enfants révoltés et parents coupables. Étude sur la désorganisation de la famille et ses conséquences sociales, Paris, A. Colin, 1895, 407 p.

(10) Évoquant la démission en 1880 d'en- son étude sur la Société générale des prisons ${ }^{(7)}$ qui fonctionne comme un lieu d'études et de rencontres. Des juristes, en utilisant les réseaux philanthropiques, tentent tantôt de contourner les problèmes rencontrés dans l'exercice de leur profession, tantôt d'œuvrer à différentes réformes pénales et sociales qui apporteraient des réponses à la question sociale.

La conviction des nouveaux philanthropes est que "l'agir" passe par la compétence et l'expérience. La profession (inspecteur des prisons, médecin, avocat) peut devenir l'assise à partir de laquelle l'engagement est jugé socialement utile. Enfant, Georges Bonjean a rencontré des "figures de transition" : son père, magistrat et visiteur de prisons, et surtout Bérenger de la Drôme, grande figure de la philanthropie libérale, fondateur en 1832 de la célèbre Société de patronage des jeunes libérés de la Seine. ${ }^{(8)}$ L'avocat, puis le juge que Georges Bonjean devient, lie son engagement philanthropique à la morale professionnelle qui l'anime. Il tire de celle-ci les leçons d'un nécessaire dévouement à la chose publique qui fait le lien entre les deux mondes dans lesquels il s'investit. Par ses idées et par ses actes, le magistrat parisien défend avec acharnement un droit au service de l'ordre, de la concorde, du bien commun. La morale prônée renvoie à des valeurs liées à la formation personnelle du juge et à sa vision de la mission que la société lui confie mais aussi à l'exercice de sa profession, comme en témoigne l'ouvrage qu'il consacre à l'application de la correction paternelle en France. ${ }^{(9)}$

La séparation des pouvoirs garantit le juste exercice de cette morale qu'il identifie à une profession. En 1879, alors que le nouveau gouvernement républicain engage une politique hostile aux congrégations religieuses qui requiert la présence de magistrats dans les procédures d'expulsion, il démissionne et redevient simple juge-suppléant. Georges Bonjean, juge catholique, proteste contre l'autoritarisme dont ses supérieurs font preuve à son égard. Contrairement à nombre de ses collègues, ${ }^{(10)}$ il fait le choix de rester dans les murs de l'institution, mais adopte volontairement une position de repli et se réfugie dans l'action philanthropique. Plus âgé, il laisse le souvenir d'un magistrat ayant l'habitude de se rendre à ses audiences au Palais de justice "en égrenant ostensiblement tout le long des quais un long rosaire dominicain ${ }^{(11)}$ ». 


\section{Morale, famille et religion}

Dans le cas particulier mais non unique de Georges Bonjean, le poids de la morale professionnelle est renforcé par l'ancrage familial. La morale est vénérée comme un héritage. Elle donne son identité à une famille qui a fait du droit un bien propre et un marqueur social. Exerçant à Paris, Georges Bonjean est un notable normand. La propriété familiale est située à Orgeville, petit village dans le département de l'Eure. Les villageois parlent du "château" et le magistrat est un propriétaire terrien.

La morale du "juste" et du "bien", comme aime à la qualifier le magistrat, s'est nourrie dans une famille d'hommes de loi : son père, comme nous l'avons déjà indiqué, a été magistrat ; ses deux frères, Maurice et Jules, sont avocats ; ses deux fils, Louis et Georges, ont également adopté la robe des avocats. La fidélité au droit dont l'exercice a accompagné l'ascension de Louis Bernard n'est pas sans lien avec la mémoire qui fait de la justice un état, propriété d'une famille. La Révolution française a aboli les offices mais l'exercice particulier de la justice, les devoirs et les honneurs qui y sont attachés, en font une profession à part, qui dépasse la rupture privé-public. L'exercice du droit fonde ici une dynastie. L'histoire de la famille Bonjean transforme un état en destinée.

Le sens donné à la morale professionnelle et le sens de l'engagement philanthropique de Georges Bonjean ont été façonnés par le traumatisme qu'a représenté l'exécution du héros familial : Louis Bernard Bonjean. ${ }^{(12)}$ La mort du père dans l'exercice de ses fonctions (fusillé en 1871 par les Communards comme président de la Cour de cassation aux côtés de Mgr Darboy) transforme la fonction en une vocation familiale. Dans les années qui suivent ces événements les descendants du "Président » célèbrent le «martyre » de celui qui, face à l'anarchie, représentait le droit. La représentation, dans la chapelle familiale, de la cellule de Mazas dans laquelle le Président a séjourné l'illustre. De même que la constitution d'une bibliothèque familiale par la veuve du défunt, dans laquelle une place importante est faite à une collection d'ouvrages sur la Commune, recouverts de noir. Autre acte de mémoire, signe d'une culture du sacrifice : en 1914, lorsque le fils aîné de Georges part à la guerre, il lui confie viron 500 magistrats refusant de participer à l'exécution des arrêtés de dissolution des congrégations religieuses non autorisées à enseigner, Jean-Pierre ROYER, Renée MARTINAGE, Pierre LECOQ décrivent " un suicide collectif ", un "phénomène d'auto-épuration unique par son ampleur ", des «bommes qui quittent le service d'un gouvernement, qui, à leurs yeux, s'est fourvoyé et agit contre le droit ", Juges et notables au XIXème siècle, PUF, 1982, pp. 376-388.

(11) Lettre de

Mme Rivet, petite fille de Georges Bonjean, à l'auteur, juillet 1991.

(12) Voir l'ouvrage de G. Peyron-Montagnon, Un dauphinois otage de la Commune. Le Président Bonjean 1804-1871, Valence, Sorepi, 1973, 257 p. 
(13) Pascale QuincyLefebvre, Enfants désespérants, enfants désespérés.

Pour une histoire de la jeunesse difficile en France, de la famille à la maison de correction (1880-fin des années 1930), mémoire de DEA sous la direction de Michelle PERROT et d'André GUESLIN, 1991, Paris 7, p. 45. un morceau du gilet ensanglanté de l'aïeul. ${ }^{(13)}$ L'acte symbolise la mission particulière à laquelle la famille s'est identifiée : servir la Nation et le Droit jusqu'au sacrifice de sa vie.

La culture familiale du souvenir, la force de l'engagement professionnel et le lien créé avec la philanthropie renforcent les convictions religieuses de Georges Bonjean. L'hommage rendu à l'ancêtre célèbre certes le défenseur de l'ordre et du droit mais aussi l'homme de Dieu présenté comme un véritable martyr de la foi. Par le pardon accordé à ses bourreaux, il est salué comme celui qui a su montrer la voie à ses héritiers : l'action charitable et sociale est seule capable de ramener dans la vérité les peuples égarés et de sanctifier ceux qui s'y consacrent. Pour évoquer la figure de défunt, sa veuve parle du "Saint". Plus d'un demi-siècle plus tard, sa petite fille, Françoise, une religieuse, reprend par d'autres biais, une partie des œuvres fondées par son père, engage une demande de béatification auprès du Saint-Siège.

Pour Georges Bonjean, la religion est une source d'inspiration du juste. La pratique du droit et l'action sociale sont des moyens d'appliquer sur terre le plan divin d'harmonie et de concorde pour lequel le Président a sacrifié sa vie. Inspirés par leur mère, lui et les siens voient dans la mort du Président un signe de Dieu à fertiliser. La mort doit permettre l'expiation des erreurs du temps. Le pardon accordé à ses "assassins" est une leçon à partir de laquelle peut se reconstruire l'harmonie perdue. L'engagement dans le droit et dans l'action sociale de ses héritiers est présenté comme relevant d'un même idéal religieux de restauration de la concorde entre les hommes. Comme l'interprétation des paroles du martyr par sa veuve les y convie, ils doivent œuvrer au salut de leurs contemporains et témoigner, par leurs actions, de la valeur religieuse de la mort du père. Le juste et le bon sont dès lors associés pour que le saint puisse être reconnu. Âgé d'une quarantaine d'années et soucieux de développer les œuvres qu'il a créées, auteur d'une brochure consacrée à la mémoire de son père, Georges Bonjean n'hésite pas à en appeler au patronage de l'ancêtre lorsqu'il écrit une nouvelle fois : " $S i$ j’ai sacrifié et moi-même et ma sainte compagne, puis mes nobles enfants, à la 
tâche de recueillir d'abord les orphelins, ensuite ces jeunes êtres en danger moral qui auraient pu constituer dans l'avenir, de nouveaux pelotons d'exécution pour de nouveaux otages, c'est au grand pardon du Président Bonjean et à ses leçons de rigoureuse austérité que j’ai obéi. » ${ }^{(1)}$

L'engagement de l'homme du droit et de l'homme d'œuvre qu'il est devenu puise ses racines dans l'interprétation d'un drame familial. La Commune est le grand crime contre l'ordre, la Nation et la Religion, à partir duquel est pensée l'action professionnelle, et plus encore l'action sociale développée par le magistrat lorsqu'il doute de la possibilité de pouvoir exercer normalement sa charge dans un État sans Dieu. Un parallèle peut être établi, même si les options politiques sont différentes, avec Jules Gossin (mort en 1855), également magistrat, fondateur en 1826 de l'œuvre de St-François Régis au service du mariage religieux des ouvriers, et dont le père avait été guillotiné pendant la Terreur, le 5 Thermidor. Catherine Duprat présente cet homme de charité, impliqué dans une œuvre ouvertement confessionnelle et missionnaire, comme une figure d'un catholicisme de reconquête ayant consacré sa vie à combattre la révolution et ses gangrènes, l'irréligion, l'esprit de révolte, la corruption des mœurs. ${ }^{(15)}$

Dans les années 1880, Georges Bonjean dit vouloir placer ses œuvres au-dessus des querelles politiques et religieuses. Il est un magistrat au service d'une République laïque et il pense que l'action philanthropique doit rassembler toutes les énergies. Mais, dans les décennies qui suivent la fondation de sa société de patronage, il manifeste plus ou moins ouvertement une forte hostilité à l'égard de maints gouvernements républicains et critique une république «ennemie de la religion ». Au cours des années 1900-1910, ses supérieurs semblent d'ailleurs douter de son républicanisme, et une certaine presse le présente comme un suppôt de la réaction cléricale. La situation est plus complexe. Ce fils d'un ancien orléaniste rallié à l'Empire est hostile au retour de la Monarchie en France. Catholique, patriote, il ne rejoint pas l'Action française qu'il condamne tout en critiquant l'orientation empruntée par Marc Sangnier. Il approuve l'appel au ralliement lancé par le pape. En fait, la forme politique lui importe assez peu pourvu que le régime puisse inté-
(14) Ibid.

(15) Georges Bonjean, Le Président Bonjean 1804-1871, Paris, 1908, p. 48 . 
(16) Pour la correspondance entre le magistrat et sa hiérarchie, voir son dossier personnel aux Archives nationales : A.N. BB 6 II 687, 716. grer et pacifier les ouvriers des villes dans le respect des valeurs du christianisme. Développant une forte activité en direction des ouvriers vers les années 1910 en lançant des projets de coopératives de production et de consommation, en construisant des logements ouvriers, il finit même par se déclarer "socialiste » : un socialiste dont l'ambition est l'amélioration du sort de la classe ouvrière afin de mieux la détourner de l'idéal révolutionnaire du mouvement ouvrier. Dans une lettre au ministre de la Justice du 13 septembre 1913, alors qu'il est suspecté par la presse radicale de financer "l'anarchie" à un moment où il cherche à pénétrer le milieu pour le pacifier, le vieil homme qu'il est devenu, amené à faire le bilan de son activité, déclare une nouvelle fois : "J'ai consacré ma vie aux questions sociales dans l'espoir de pacifier les milieux révolutionnaires. ${ }^{(16) ~ " ~}$

Homme de convictions, Georges Bonjean aimait que l'on voit en lui un homme de devoir. Magistrat et philanthrope, il entendit bâtir sur terre des cités harmonieuses loin des périls révolutionnaires. Homme de combat, il partit en guerre contre le désordre. Ses armes furent le droit et le social mis au service d'une morale d'ordre à travers laquelle peut se comprendre le type social du magistrat-philanthrope de la deuxième moitié du XIXème siècle.

\section{Le magistrat-philanthrope : acteur de la protection de l'enfance}

Par les qualités qui lui sont associées sur le plan de la morale sociale ou privée, l'engagement philanthropique de certains hommes de loi définit un rôle et confirme leur appartenance à l'élite. La "cause" se nourrit de la fréquentation de certains lieux qui font explicitement le pont entre les deux univers que sont la Société générale des prisons et les comités de défense des enfants traduits en justice qui se créent, à partir de 1890, dans quelques grandes villes françaises. Des idéaux communs se manifestent par l'attachement à l'ordre et au juste, par le souci du bien commun, mais aussi par l'adhésion aux valeurs républicaines de solidarité. Cependant, l'implication de nombreux hommes de loi dans la construction du social par le biais de la philanthropie peut être interprétée comme une manière détournée de faire de la politique lorsque leurs idées ne rencontraient pas celles du pouvoir. Ce fut le cas pour de nombreux libéraux sous la Restauration 
mais aussi de catholiques conservateurs sous la Troisième République. Lieu d'expérience et plate-forme pour des propositions, la philanthropie ne fut pas sans relation avec la justice civile et pénale dans la France de la IIIème République, construisant le futur secteur de l'enfance inadaptée. Avec Henri Rollet et Louis Albanel, Georges Bonjean a participé à la réforme de la justice et à la mise en place d'une protection des mineurs en France.

\section{Philanthropie et délinquance des mineurs}

Comme beaucoup de ses collègues de la même époque, Georges Bonjean appartenait à la famille des juges qui firent preuve d'un "scepticisme carcéral viscéral" (17) lorsque le délinquant était un mineur. Il avait certes confiance dans les qualités répressives de la prison, mais il ne croyait pas qu'elle puisse être l'école du pauvre et résoudre la question sociale. Libéral, il plaçait ses espoirs dans l'initiative privée pour "la réforme des jeunes délinquants ». De la sorte, il se situait dans l'esprit de la loi du 5 novembre 1850, qui invitait les personnes privées à fonder des colonies agricoles pour accueillir les mineurs acquittés comme ayant agi sans discernement mais devaient bénéficier d'une éducation correctionnelle jusqu’à leur majorité. La plus connue était alors la colonie de Mettray ouverte sous la monarchie de juillet, par un magistrat, F. A. Demetz. ${ }^{(18)}$ Sur ce modèle, dans une première étape, Georges Bonjean fonda la colonie agricole d'Orgeville sur ses terres. Elle fut d'abord ouverte pour accueillir des orphelins en 1874. Puis, à partir de 1877 et jusqu'en 1882, elle reçut des jeunes délinquants confiés par l'administration pénitentiaire. ${ }^{(19)}$

Dans la deuxième moitié du XIXème siècle, la floraison de colonies agricoles, à l'initiative de philanthropes dont certains étaient des magistrats, eut des implications directes dans la justice des mineurs. L'usage de l'article 66 du Code pénal devenait plus fréquent. Il permettait l'enfermement des mineurs sans les envoyer en prison. A la place de la peine de quelques mois dans une maison d'arrêt et de correction, où des quartiers spécifiques existaient très rarement, il autorisait l'envoi, dans une colonie pénitentiaire, de mineurs jusqu'à leur majorité. Corriger, réformer, moraliser devenaient les mots d'ordre à partir desquels l'initiative philanthropique était reconnue et soutenue dans le prétoire.
(17) Voir Robert

BADINTER, La prison

républicaine, Paris,

Fayard, 1992, 430 p.

(18) A la différence de Georges Bonjean, une fois la colonie créée,

Demetz démissionna de ses fonctions de magistrat pour se consacrer entièrement à son œuvre. Voir les travaux de Jacques BOURQUIN et d'Éric PIERRE.

(19) Pascale QUINCYLEFEBVRE, Familles, institutions et déviances. Une histoire de l'enfance difficile 1880-fin des années 30, Paris, Economica, 1997. 
(20) Très vite, devait

d'ailleurs éclater une querelle en paternité entre le magistrat-philanthrope et l'administrateur de l'Assistance publique, Loys Brueyre.

(21) Pierre GUILLAU-

$\mathrm{ME}$, Un siècle d'histoire de l'enfance inadaptée, l'OREAG, 1889-1989, Paris, Expansion scientifique française, 1989 , $120 \mathrm{p}$.

(22) Dominique DESSERTINE, La Société lyonnaise pour le sauvetage de l'enfance, 1890-1960. Face à l'enfance en danger, un siècle d'expérience de l'internat et du placement familial, Toulouse, Erés, 1990, 218 p.

\section{Philanthropie et enfance en danger moral}

Avec la création de la colonie d'Orgeville, le juge Bonjean se situait donc dans une tradition déjà bien établie. En son temps, il fit pourtant preuve de plus d'innovation. Dans le sillage d'un mouvement général qui plaçait la notion de prévention au cour de la question sociale, il créa la Société générale de protection pour l'enfance abandonnée ou coupable. Par ce biais, il entendait faire la promotion de la rééducation, par l'initiative privée, d'un public de mineurs présenté comme moralement abandonné ou en danger moral et pouvant basculer dans la délinquance. Le projet prenait le contre-pied du scepticisme judiciaire ambiant et croyait à la possibilité de réformer des jeunes ayant déjà passé à l'acte. Il convenait également de mener un travail de prévention auprès d'une population à risque. A l'époque, à côté de l'Assistance publique, exista un mouvement d'opinion qui aboutit, avant même l'adoption de la loi de 1889 sur la déchéance paternelle, à la création d'un service des enfants moralement abandonnés dans le département de la Seine. ${ }^{(20)}$ Dans les deux décennies qui suivirent, l'exemple de Georges Bonjean fut repris. En 1888, fut fondée l'Union française pour le sauvetage de l'enfance ; en 1900, l'avocat Henri Rollet prit l'initiative de mettre sur pied le Patronage de l'enfance et de l'adolescence. Vers la même époque sont créées une association bordelaise, étudiée par Pierre Guillaume ${ }^{(21)}$ dont les fondateurs font explicitement référence à la société imaginée par Georges Bonjean et une association lyonnaise, étudiée par Dominique Dessertine. ${ }^{(22)}$

Les sociétés de patronage fonctionnaient comme des centres de placement d'enfants, dans des familles rurales, quelquefois dans des ateliers urbains, plus fréquemment dans des établissements baptisés colonies agricoles, écoles de réformes ou de préservation. De telles institutions étaient finalement peu différentes des colonies pénitentiaires de l'époque. Les sociétés de patronage étaient aussi des lieux de réflexion sur les questions pénitentiaires et de protection de l'enfance. En 1883, par exemple, la société fondée par Georges Bonjean organisa un congrès international de la Protection de l'enfance. Certaines de ces sociétés développèrent des actions de "lobbying" auprès de personnalités politiques pour soutenir 
tel ou tel projet de loi. Ainsi, en 1879, La société Bonjean et la publicité faite autour de ses premières réalisations n'étaient pas sans lien avec le combat législatif du sénateur Théophile Roussel pour faire passer un projet de loi sur la déchéance paternelle.

Le juge Bonjean fut capable d'animer des réseaux, de rassembler des fonds, de mobiliser l'opinion pour que la situation des enfants moralement abandonnés soit prise en compte. Même si la législation sur la déchéance paternelle fut peu appliquée sa seule existence transforma en profondeur la pratique judiciaire des tribunaux. La loi de 1850 sur l'éducation correctionnelle, les lois de 1889 (déchéance), de 1898 (mauvais traitements à enfants), de 1912 (Création des tribunaux pour enfants et adolescents, liberté surveillée), les décrets-loi d'octobre 1935 sur la correction paternelle et l'assistance éducative dessinèrent une nouvelle justice et placèrent les milieux philanthropiques et les premiers services sociaux sur le devant de la scène. En effet, la collaboration public-privé ne fut pas sans nuage et suscita de vives critiques. ${ }^{(23)}$

Le magistrat philanthrope est une figure à retenir dans une histoire sociale et culturelle de la magistrature et plus largement dans une histoire de la justice. Par sa position à la charnière des sphères privé-public, avant la professionnalisation du social, il a été un passeur d'idées actif dans la construction du social.

Le magistrat-philanthrope est un acteur reconnu par l'opinion et par l'administration au XIXème. Au début du XXème siècle, alors que s'éteint un certain âge de la philanthropie, il est de plus en plus critiqué dans l'exercice de ses fonctions et de ses engagements respectifs. Ainsi, Georges Bonjean, lorsqu'il devient juge titulaire en 1894, bénéficie de la part de ses supérieurs qui saluent son dévouement, d'un rapport favorable Mais plus tard, en 1909-1910, lorsque la bonne gestion de ses œuvres est mise en cause, que son nom et sa fonction sont mêlés à des "affaires" reprises par la presse, il doit affronter une hiérarchie sensible aux effets du scandale sur l'image de la profession. La réaction de ses supérieurs devient particulièrement vive lorsque le magistrat élargit son action sociale en direction du monde ouvrier. Mais ils ne sont pas les seuls. En 1911, le journal radical L'Action le décrit comme « un conspira-

(23) A plusieurs reprise, des dysfonctionnements éclatèrent au grand jour dans la vie des ouvres et dans le fonctionnement des premiers TEA, et des crises mobilisèrent l'opinion autour de la critique de la figure ambigüe du juge-philanthrope accusé par ses décisions d'homme de loi de pourvoir aux besoins des œuvres qu'il animait. A propos des difficultés du juge

Henri Rollet, voir Monique CHARVIN, Jean-François GAZEAU, Eric PIERRE, Françoise TÉTARD, Recherche sur les juges des enfants.

Approche historique, démographique, sociologique,

Rapport final, conseil de la recherche du ministère de la Justice, 1996 ; voir aussi aussi l'ouvrage de Paul BERTRAND, Monsieur Rollet, le "dernier des philantbropes", Paris, PUF, publications du CTNERHI, 1986, 282 p. 
(24) Contraction de "papa-fondateur".

(25) A.N. BB 6 II 687, 716. teur en robe, tourmenté de rage obscure, hanté par la folie des grandeurs, sans cesse à l'affut d'une ouvre à compromettre, pirouettant au milieu des efforts contradictoires et de contresens comme une toupie ivre ". En 1913, dans Les hommes du jour, en 1913, deux grands articles de Victor Méric stigmatisent ce " Magistrat et négociant en philanthropie " et "ce cher papa-dateur ${ }^{(24)}$ qui aime ses enfants à la façon dont l'ogre aime le petit poucet et ses frères". L'administration est sommée de réagir par une presse radicale et socialiste en colère. L'Action du 12 janvier 1913 titre : "Un scandale permanent : le juge Bonjean subventionne l'anarchie et l'antimilitarisme. Qu'attend la Cour de Cassation?" Les autorités concernées semoncent alors le "provocateur” qui en appelle à l'intérêt social supérieur de ces œuvres destinées à détourner l'ouvrier des associations de "malfaiteurs " pourvoyeuses du "bluffprolétarien ». Les explications ne convainquent guère. Dans un rapport interne, un président du tribunal de la Seine dénonce les dangers que représente, pour la magistrature, cet homme qui se prend pour « un apôtre " mais qui n'est "qu'une honteuse mais incorrigible dupe ". ${ }^{(25)}$

Confrontée à d'autres scandales médiatiques mettant en cause des figures de juges-philanthropes, comme lors de l'affaire Rollet, pendant l'entre-deux-guerres, la hiérarchie invite la magistrature à plus de prudence. A partir des années trente et quarante, les profondes transformations qui affectent la protection judiciaire de la jeunesse en construction redéfinissent le rôle des juges et leur relation avec le monde associatif. Un nouveau paysage institutionnel, qui mise sur une étroite collaboration entre le privé et le public, s'impose alors. 
Comment désobéir à l'ordre magnanime

Dicté par un héros! Les fils ont pardonné

A l'exemple divin du Rédempteur sublime

Priant pour les ingrats qui l'avaient condamné !

Refoulant en son cœur tout ferment de vengeance.

L'aîné, vraiment chrétien, pouvant plus faire émoi,

Résolut d'adopter la malheureuse engeance

De ces grands criminels, par Dieu punis de mort!

Bientôt, on vit s'ouvrir une maison nouvelle

Qui abrite le tombeau de son père martyr ;

c'est là qu'il ébauche son œuvre paternelle

Dont les progrès, dès lors, n'ont cessé de grandir.

En voyant se mouvoir dans cette ruche agreste

Un essaim florissant de travailleurs joyeux,

Aux traits épanouis, à l'allure si leste.

Dirait-on que jamais ils furent malheureux ?

Dieu des infortunés! montrez-vous favorable

Aux généreux desseins de cet homme de cœur

Fondez le succès de son plan secourable

Pour ces prédestinés du crime et du malheur. ${ }^{(26)}$

(26) Extrait d'un poème écrit à la fin du

XIXème siècle par

Adélaïde Marie Bonjean, épouse de Louis Bernard

Bonjean et mère de

Georges Bonjean évo-

quant la création de la

colonie agricole

d'Orgeville (A.D. de

l'Eure 40 J 26. Dossier

du fonds privé déposé

par Melle Lanquetin,

dernière directrice

d'Orgeville. Cahier 1 bis.

Le président et la Présidence :

la Présidence Bonjean et

Appendices divers.) 